\title{
Electrochemical Nucleation, Growth and Dendrite Formation in Liquid Cell TEM
}

\author{
F. M. Ross
}

\section{IBM T.J. Watson Research Center, Yorktown Heights, NY 10598}

The relationship between the morphology of electrodeposited materials and the conditions under which they are formed is a key issue for microelectronics, where interconnects are deposited electrochemically, and for batteries, where improvements in energy storage and delivery are required. For advanced interconnects, copper deposited on a dissimilar substrate such as ruthenium must form a continuous layer with as low a thickness as possible. This requires an understanding of copper nucleation, growth and coalescence in the presence of the complex electrolyte chemistry. In the case of batteries, the electrode morphology changes on every charge/discharge cycle as the anode material goes into solution and is then redeposited. Deposition in the form of a dense, compact layer, rather than a porous or dendritic layer, is essential if the battery is to be cycled repeatedly without significant differences in performance. Changes in electrode surface area affect the charging efficiency and the current the battery can supply, and performance may be seriously degraded if, for example, dendrites bridge the electrolyte and short out the battery.

To investigate electrochemical processes such as these, we need to correlate the electrochemical parameters with structural information obtained as electrodeposition and stripping take place. This requires sub-second time resolution and reasonable spatial resolution (at least tens of nanometers) in the liquid environment. We have therefore developed a liquid cell for the transmission electron microscope that allows electrochemical control of a process while under electron beam observation $[1,2]$. Electrolyte is enclosed between silicon nitride windows, and three electrodes are integrated within the cell to allow full electrochemical control. The liquid thickness is of the order of $1 \mu \mathrm{m}$ and depends on the designed spacing between the windows and the bowing of the windows in the microscope vacuum. A transparent working electrode extends over the window while the counterand reference electrodes are out of view. Images are recorded at $300 \mathrm{kV}$ under bright field conditions, and zero-loss energy filtering may be used to improve contrast through the thick specimen.

Processes occurring on the working electrode can be observed in plan view geometry. This is ideal for quantitative observations of nucleation and growth (FIG. 1a, b). We have examined copper growth on polycrystalline $\mathrm{Au}$ or $\mathrm{Ru}$ from acidified copper sulphate solutions [2-4]. Nucleation is progressive, and the growth exponents of individual nuclei follow the predictions of diffusion limited models; however, quantitative comparison suggests that surface diffusion should be included to understand fully the nucleus density and growth rates. In the microelectronics context, growth is modified through the use of additives in the electrolyte, and an understanding of additive effects is essential for controlling copper structure. In situ experiments show clear differences in nucleus shape and distribution (FIG. 1c, d), but we find that the electron beam can have a significant effect, perhaps because some of the additives are organic molecules [5].

The processes taking place during battery cycling may be visualized by observing growth at the edge of the working electrode. In FIG. 2, we show dendritic growth of copper obtained galvanostatically at high current density. In situations such as this we find that dissolution occurs from the base of the dendrites as well as the tips. As soon as the base is etched through, the tip detaches and floats away 
in the solution. Quantifying the conditions under which this type of process takes place, for example for zinc or lithium, may be useful in understanding battery performance.

Electrochemical liquid cell TEM can provide a unique window into a wide range of important processes. Once electrochemical control is combined with the recently available liquid flow cells [7], even further exciting opportunities may become possible [8].

\section{References}

[1] M. J. Williamson, R. M. Tromp, P. M. Vereecken, R. Hull and F. M. Ross, Nature Mater. 2 (2003) 532.

[2] A. Radisic, P. M. Vereecken, J. B. Hannon, P. C. Searson and F. M. Ross, Nano Lett. 6 (2006) 238.

[3] A. Radisic, P. M. Vereecken, P. C. Searson, and F. M. Ross, Surf. Sci. 600 (2006) 1817.

[4] A. Radisic, F. M. Ross and P. C. Searson, J. Phys. Chem. B 110 (2006) 7862.

[5] M. den Heijer, In-situ transmission electron microscopy of electrodeposition: Technical development, beam effects and lithography, M. Sc. Thesis, Leiden University (2008)

[6] http://www.hummingbirdscientific.com/PdfFiles/LiquidCellHolder.pdf

[7] We acknowledge M. C. Reuter, A. W. Ellis, S. J. Chey, A. Prabhakar and R. Sirdeshmukh for their technical contributions to this project, P. C. Searson, P. M. Vereecken and I. Shao for electrochemical insights, and M. J. Williamson, A. Radisic and M. den Heijer for the experimental work.
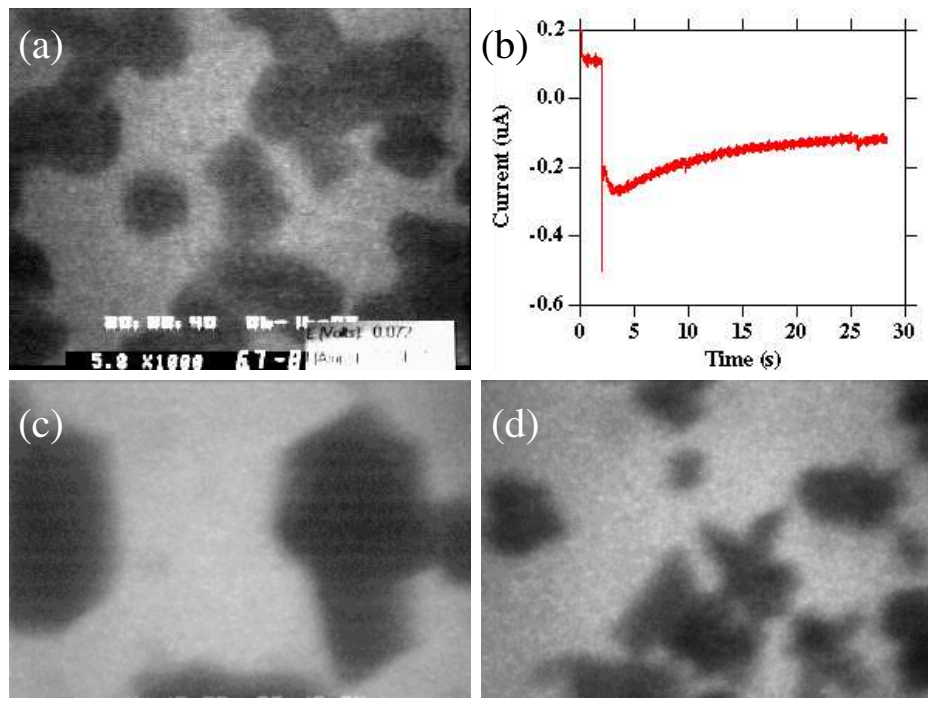

FIG. 1. Bright field images recorded at $300 \mathrm{kV}$ during deposition of copper in situ. (a) Rounded nuclei formed on polycrystalline $\mathrm{Au}$ from $0.1 \mathrm{M} \mathrm{CuSO}_{4}+0.1 \mathrm{M}$ $\mathrm{H}_{2} \mathrm{SO}_{4}$ at a potential of $-0.07 \mathrm{~V}$ with respect to $\mathrm{Cu}$, shown after 25 seconds; (b) Current versus time recorded during this deposition; (c) Faceted crystals on polycrystalline $\mathrm{Ru}$ deposited from $0.63 \mathrm{M}$ $\mathrm{CuSO}_{4}+0.1 \mathrm{M} \mathrm{H}_{2} \mathrm{SO}_{4}+1.4 \mathrm{mM} \mathrm{HCl}$ at $-100 \mathrm{mV}$; (d) Needle shaped crystals from the same electrolyte at $-0.5 \mathrm{~V}$ but with suppressor and leveler additives. The horizontal field of view is $1.8 \mu \mathrm{m}$.

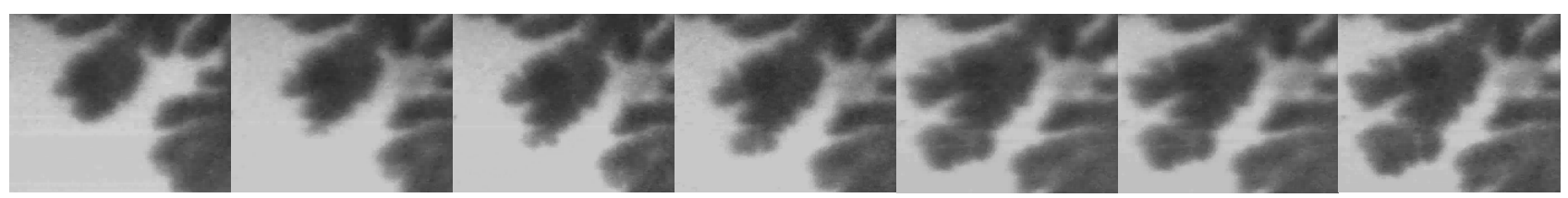

FIG. 2. Series of bright field, energy filtered images extracted from a video recorded in situ showing the growth of dendritic arms of copper from the edge of a polycrystalline Au electrode. The electrolyte was $0.1 \mathrm{M} \mathrm{CuSO}_{4} / \mathrm{H}_{2} \mathrm{SO}_{4}$ and growth conditions were galvanostatic with $40 \mathrm{mAcm}^{-2}$ (1 $\mathrm{mA}$ total current). Images are shown at 8 second intervals. The field of view is $6.25 \mu \mathrm{m}$. 